\title{
Erosion and deposition in depth-averaged models of dense, dry, inclined, granular flows
}

\author{
James T. Jenkins ${ }^{1}$ and Diego Berzi ${ }^{2}$ \\ ${ }^{1}$ Cornell University, Ithaca, New York 14853, USA \\ ${ }^{2}$ Politecnico di Milano, 20133 Milano, Italy \\ (Received 15 July 2016; published 8 November 2016)
}

\begin{abstract}
We derive expressions for the rates of erosion and deposition at the interface between a dense, dry, inclined granular flow and an erodible bed. In obtaining these, we assume that the interface between the flowing grains and the bed moves with the speed of a pressure wave in the flow, for deposition, or with the speed of a disturbance through the contacting particles in the bed, for erosion. We employ the expressions for the rates of erosion and deposition to show that after an abrupt change in the angle of inclination of the bed the characteristic time for the motion of the interface is much shorter than the characteristic time of the flow. This eliminates the need for introducing models of erosion and deposition rate in the mass balance; and the instantaneous value of the particle flux is the same function of the instantaneous value of the flow depth as in a steady, uniform flow.
\end{abstract}

DOI: 10.1103/PhysRevE.94.052904

\section{INTRODUCTION}

Many granular flows in nature take place over erodible beds; such flows are also studied in laboratory experiments [1-4], and numerical simulations [1,5]. When the flows are unsteady or developing, erosion from the bed or deposition onto it will, in general, occur. The question, then, is how to model these phenomena. The simplest case is when the motion of the interface between the bed and flow is known, as in experiments in a rotating drum [6,7]. In this case, the rate at which material enters or leaves the flow is known and the stress ratio at the surface of the bed has its yield value $[8,9]$. The more complicated situation is when the motion of the interface must be determined. Existing models for its determination [10-15] differ.

Capart and Young [11] assume that the velocity of the interface relative to the material is proportional to the difference between the instantaneous and the steady, uniform particle fluxes; Fraccarollo and Capart [12] and Iverson [15] assume that it is proportional to the ratio of the jump in shear stress across the interface to that of tangential momentum, with an immediate adjustment in flow velocity; Naaim et al. [13] assume it to be proportional to the difference between the tangent of the angle of inclination and the stress ratio at start or at stop; Bouchaud et al. [10] and Tai and Kuo [14] assume that it is proportional to the difference between the angle of inclination and the neutral angle.

Here, we assume that the motion of the interface between the flow and the bed is determined by a wave speed in the bed, for erosion, and by a wave speed in the flow, for deposition. That is, we assume that the top of the bed is the interface between ephemeral, unconnected chains of colliding spheres of the flow and more permanent, connected chains that transmit force downward through the aggregate of less agitated grains of the bed.

The speed of propagation of this interface depends on the speed of disturbances that make or break the force chains of the bed. We provide explicit expressions for these wave speeds and calculate them in simple situations. We assume that the shear stress and tangential momentum are continuous across the interface, but permit a jump in the component of particle velocity perpendicular to the interface.
We employ this information to calculate the response of an initially steady and uniform flow to an abrupt, uniform change in the angle of inclination of the bed. This is the situation considered by Jop et al. [16] in their experiments. An explicit calculation of the time scales associated with the motion of the interface for erosion and deposition indicates that they are short compared to the time of adjustment of the depth-averaged flow. This ensures that the ratio of shear stress to normal stress at the base of the flow, given in terms of the depth of the flow and the depth-averaged velocity in a steady, uniform flow, is equal to the yield stress ratio. Using this, the depth-averaged flow momentum balance alone determines the time evolution of the flow in the Jop et al. experiments.

\section{GOVERNING EQUATIONS}

We consider steady, uniform flows of dense aggregates of identical spheres. In order for a steady, uniform flow over an erodible bed to exist, it must be resisted by frictional sidewalls. Such sidewalls induce variations between the walls. We initially focus on the interface between the flow and the bed and ignore the variations in flow quantities induced by the walls.

\section{A. Micromechanics}

The collisional interaction between pairs of particles is characterized through the coefficient of normal restitution $e$, the ratio of the magnitudes of their relative velocity along their line of centers after and before a collision; interactions through enduring contacts are characterized in terms of the material shear modulus $S$ and Poisson's ratio $\psi$. The influence of sliding friction and tangential restitution in collisions may be incorporated through an effective coefficient of restitution [17].

We assume that the top of the bed is the interface between the ephemeral, unconnected chains or clusters of colliding spheres of the flow and more permanent, connected chains that transmit force downward through the aggregate of the less agitated grains of the bed. At this interface, we assume that the ratio of particle shear stress $s$ to the particle pressure $p$ has a characteristic value [18]. We refer to this value as the yield 
stress ratio $\alpha$; it is determined by contact elasticity and friction and the geometry of the packing. The location of the interface changes as value of the stress ratio at the bottom of the flow changes. We focus here on the change in the stress ratio due to a change in pressure. Changes in pressure communicated through the flow or through the bed influence the stress ratio and the location of the bed.

The motion of the interface is determined by the balance of mass and momentum across it. The balance of mass at a surface of discontinuity that moves with velocity $\mathbf{c}$ through a material with velocities $\dot{\mathbf{x}}^{+}$and $\dot{\mathbf{x}}^{-}$and mass densities $\rho^{+}$and $\rho^{-}$on the front and back of the surface is

$$
\left(\rho^{+} U_{i}^{+}-\rho^{-} U_{i}^{-}\right) n_{i}^{-}=0,
$$

where $\mathbf{U}^{ \pm} \equiv \mathbf{c}-\dot{\mathbf{x}}^{ \pm}$and $\mathbf{n}^{-}$is the unit outward normal to the region behind the surface. Similarly, the balance of linear momentum across the surface is

$$
-\left(\rho^{+} \dot{x}_{i}^{+} U_{k}^{+}-\rho^{-} \dot{x}_{i}^{-} U_{k}^{-}\right) n_{k}^{-}=\left(t_{i k}^{+}-t_{i k}^{-}\right) n_{k}^{-}
$$

where $\mathbf{t}^{+}$and $\mathbf{t}^{-}$are the stress tensors at the front and back of the surface.

For erosion, the wave in the bed is associated with a reduction of compression, $\dot{\mathbf{x}}^{-} \cdot \mathbf{n}^{-}<0$, and there is a discontinuity in the normal component of the velocity at the front. In this case, the wave propagates with velocity $\mathbf{c} \cdot \mathbf{n}^{-}>0$ into material at rest, $\dot{\mathbf{x}}^{+} \cdot \mathbf{n}^{-}=0$; so $\mathbf{U}^{+} \cdot \mathbf{n}^{-}=\mathbf{c} \cdot \mathbf{n}^{-}$and $\mathbf{U}^{-} \cdot \mathbf{n}^{-}=\left(\mathbf{c}-\dot{\mathbf{x}}^{-}\right) \cdot \mathbf{n}^{-}$is positive. Then,

$$
\rho^{+} c_{i} n_{i}^{-}=\rho^{-}\left(c_{i}-\dot{x}_{i}^{-}\right) n_{i}^{-}
$$

and

$$
\rho^{-} \dot{x}_{i}^{-} n_{i}^{-}\left(c_{k}-\dot{x}_{k}^{-}\right) n_{k}^{-}=n_{i}^{-}\left(t_{i k}^{+}-t_{i k}^{-}\right) n_{k}^{-} .
$$

That is, the change in momentum normal to the surface of the bed is equal to the discontinuity in the normal component of the stress across the wave. Parallel arguments applied to deposition lead to the same conclusion.

In a recent paper, Iverson [15] adopts the approach of Fraccarollo and Capart [12] and relates the rate of erosion and deposition to the ratio of assumed discontinuities in the tangential components of the tractions and the particle velocities at the interface between the flow and the bed. This permits the mass density to remain continuous across the interface and, if the speed of propagation of the interface is somehow specified, Eq. (2) gives the difference in the flow velocity across the interface. However, to our knowledge, no such discontinuity in flow velocity is observed across the moving surface of the bed.

We assume that the pressure, not the shear stress, is discontinuous. Then, given the mass density ahead of the interface, the discontinuity in pressure across it, and the speed of the interface, the mass and momentum balances, Eqs. (1) and (2), determine, respectively, the mass density and the normal particle velocity behind the interface. Discreteelement numerical simulations of uniform, time-dependent flows of spheres between frictional sidewalls should be able to distinguish between these two very different models for the motion of the surface of a particle bed. We next relate the speed of the interface to the physics of the flow and the bed in the simplest possible way.
For erosion, we determine the speed of the pressure wave in the bed from moduli calculated by Digby [19] and Walton [20], who considered a random aggregate of identical, frictional spheres compressed by an average pressure $p$ in which the distribution of contacts was isotropic. They assumed that the relative displacement of the centers of two contacting particles was given by the average strain, and obtained expressions for the effective shear modulus $S^{E}$ and bulk modulus $B^{E}$ :

$$
S^{E}=\frac{Z v}{5 \pi} \frac{S}{1-\psi}\left(\frac{3 \pi}{2} \frac{1-\psi}{Z v} \frac{p}{S}\right)^{1 / 3} \frac{2-\psi+3 \xi(1-\psi)}{2-\psi}
$$

and

$$
B^{E}=\frac{Z v}{3 \pi} \frac{S}{1-\psi}\left(\frac{3 \pi}{2} \frac{1-\psi}{Z v} \frac{p}{S}\right)^{1 / 3},
$$

where $Z$ is the average number of contacts per particle (the coordination number) and $v$ is the solid volume fraction. The parameter $\xi$ describes the strength of the transverse stiffness of the grain-to-grain contact; $\xi=0$ for frictionless interactions (perfect slip) and $\xi=1$ for fully frictional interactions (perfect stick). The value of the bulk modulus agrees well with those measured in numerical simulations, while the value of the shear modulus is as much as three times higher [21]. The speed $c$ of the pressure wave in the bed is given in terms of these by

$$
c^{2}=\frac{B^{E}+2 S^{E}}{\rho_{s} v},
$$

where $\rho_{s}$ is the mass density of the material of the spheres. In adopting this, we ignore the anisotropy associated with the stress chains in the bed.

For deposition, we take the speed of the pressure wave in the flow to be the isothermal sound speed in a dense gas:

$$
c^{2}=\frac{1}{\rho_{s}} \frac{\partial p}{\partial v}
$$

where kinetic theory [22] gives

$$
p=2(1+e) \rho_{s} v G T,
$$

in which $T$ is one-third the mean square of the particle velocity fluctuations, the granular temperature, and, for dense flows [18],

$$
G=\frac{0.63 v}{0.60-v}
$$

We next employ these speeds in simple depth-averaged equations to illustrate the relationship between the time scales that are associated with the equilibration of the bed and the equilibration of the flow. The derivation of averaged equations that we employ can be extended to incorporate variations across the flow and more complicated treatment of the depth-averaged inertia. In what follows, we make lengths dimensionless by the particle diameter $d$, velocities dimensionless by $(g d)^{1 / 2}$, with $g$ the gravitational acceleration, and stresses dimensionless by $\rho_{s} g d$.

We take $x$ and $y$ to be the direction parallel and perpendicular to the flow, respectively, and assume that the flow takes place at an angle $\phi$ to the horizontal. Then, the location of the free surface is $y=h(x, t)$, where $t$ is the time, and the location 
of the erodible bed is $y=b(x, t)$. We take into account the influence of the sidewalls, at distance $W$ apart, in an average way, using their coefficient of sliding friction $\mu_{w}$. The local mean velocity of the particles has components $u$ and $v$ along $x$ and $y$. We assume that $u$ vanishes at the bed. Given that we focus on dense flows, we also assume that the local volume fraction $v$ is uniform throughout the flow.

\section{B. Depth-averaged equations}

We adopt simple depth-averaged equations for the balance of mass and flow momentum for a dense, shallow flow [23]. An overbar indicates quantities that are depth averaged over the thickness of the flow, $h-b$, and a subscript $b$ indicates a quantity evaluated at the bed, $y=b$. The slope of the center line of the flow is the average of the slopes of the upper and lower surface; we identify this slope with the average tangent of the angle $\phi$ between the flow and the horizontal. We also assume that the normal stresses are isotropic.

The depth-averaged mass balance is

$$
\frac{\partial(h-b)}{\partial t}+\frac{1}{v} \frac{\partial q}{\partial x}=v_{b}^{F}-\frac{\partial b}{\partial t},
$$

where $q$ is the particle flux (the product of volume fraction, flow depth, and depth-averaged velocity), $v_{b}^{F}$ is the $y$ component of the particle velocity at the base, in the flow, and the right-hand side of Eq. (11) is the source term associated with erosion from or deposition to the bed. Similarly, the depth-averaged flow momentum balance is

$$
\begin{aligned}
\frac{1}{v} \frac{\partial q}{\partial t}= & -\frac{1}{v^{2}} \frac{\partial}{\partial x}\left[\frac{q^{2}}{(h-b)}\right]-\frac{1}{2} \frac{\partial\left[(h-b)^{2}\right]}{\partial x} \cos \phi \\
& +(h-b) \sin \phi-\frac{p_{b}^{F}}{v} \frac{\partial b}{\partial x} \\
& -\frac{s_{b}}{v}-\frac{\mu_{w}}{W}(h-b)^{2} \cos \phi,
\end{aligned}
$$

where, in the first and last terms on the right-hand side, we have made the approximation $\bar{p}=v(h-b) \cos \phi / 2$, and $p_{b}^{F}$ is the pressure at the base, in the flow. The last two terms of Eq. (12) are, respectively, the resistance of the shear stress, $s_{b}$, at the base of the flow and that of the frictional sidewalls [2].

We require closures for the rate of erosion or deposition in the mass balance and the basal shear stress and pressure in the momentum balance.

\section{Interfacial stresses}

We assume that at the interface between the base of the flow and the surface of the bed the shear stress is continuous, but the pressure is not. In order to calculate the difference in pressure across the interface, we assume that the balances of momentum and the relation between the depth-averaged velocity, inclination, and flow thickness have the same form as in a steady, uniform flow. However, because the instantaneous, local values of the inclination and thickness differ, in general, from the steady, uniform values, so do the stresses and depth-averaged velocity. In particular, in unsteady, nonuniform situations, the ratio of the shear stress to the pressure at the base of the flow is not, in general, equal to the yield stress ratio at the bed. The associated discontinuity in pressure drives the motion of the interface and the erosion or deposition that results from its motion. This scenario assumes that, in response to a change of inclination, there is a very rapid adjustment through the depth of the flow, so that the depth-averaged flow variables are related in the same way, but do not have the same values, as in a steady, uniform flow. This is analogous to the assumption of local equilibrium made when deriving the constitutive relations in kinetic theory.

\section{Shear stress}

As indicated in the Appendix, using the steady, uniform momentum balances and the constitutive relations of extended kinetic theory [24], the basal shear stress for dense, inclined flows over erodible beds can be expressed in terms of particle flux and thickness by

$$
s_{b}=\mu_{b} p_{b}^{F},
$$

where $p_{b}^{F}$ is the pressure at the base of the flow and

$$
\mu_{b}=\frac{2^{3 / 2} \tilde{G}^{1 / 2} J}{(\pi \cos \phi)^{1 / 2}(1+e)^{1 / 2}} \frac{q}{v(h-b)^{5 / 2}}-\frac{2}{7} \mu_{w} \frac{h-b}{W},
$$

with $J=(1+e) / 2+(\pi / 4)(3 e-1)(1+e)^{2} /[24-(1-$ $e)(11-e)]$ and

$$
\tilde{G}=\left\{\frac{192 J^{2}(1-e)}{25 \hat{c} \pi^{3 / 2}(1+e)^{2}} \frac{8}{\left[2 \alpha+\mu_{w}(h-b) / W\right]^{3}}\right\}^{3},
$$

in which $\hat{c}$ is a material coefficient of order 1 , taken here to be 0.5 [18]. In the extended kinetic theory, multiple or repeated collisions begin to occur when the stress ratio is less than a value $k$ [18]. This value depends only on the coefficient of restitution:

$$
k=\left[\frac{24 J(1-e)}{5 \pi(1+e)}\right]^{1 / 2},
$$

and provides an upper limit for $\tan \phi$ for the validity of Eq. (14). Above this value, a less dense, diffusive, collisional flow is expected to occur [28].

\section{Pressures}

At the top of the bed, the particles are at yield, so that

$$
\frac{s_{b}}{p_{b}^{B}}=\alpha,
$$

where $p_{b}^{B}$ is the pressure at the top of the bed. Then, when the shear stress is assumed to be continuous across the interface, and using Eq. (13), there is the difference in pressure

$$
p_{b}^{F}-p_{b}^{B}=p_{b}^{F}\left(1-\frac{\mu_{b}}{\alpha}\right) .
$$

When $\mu_{b}$ is different from $\alpha$, the motion of the interface is driven by this pressure difference. We assume that the pressure ahead of the wave is hydrostatic: for erosion, $p_{b}^{B}=$ $v(h-b) \cos \phi$, and Eq. (18) permits the evaluation of $p_{b}^{F}$; for deposition, $p_{b}^{F}=v(h-b) \cos \phi$, and Eq. (18) permits the evaluation of $p_{b}^{B}$. With the difference in pressure across the moving discontinuity given by Eq. (18), the speed of the interface determined by Eq. (7) or Eq. (8), and the value of the 
mass density ahead of the wave known, the jump conditions Eqs. (1) and (2) give the mass density and the component of particle velocity perpendicular to the interface behind the jump.

\section{Erosion rate}

When $\mu_{b}$ exceeds $\alpha$, a rarefaction wave propagates into the bed to equilibrate the pressure difference across the interface. That is, the flow responds by increasing its thickness and eroding particles from the bed. In this case, the rate of change of $b$ in Eq. (11) is equal to the negative of the speed Eq. (7) of the pressure wave, evaluated at $y=b$. Assuming perfect slip, this has the dimensionless form

$$
\frac{\partial b}{\partial t}=-\left(\frac{11}{15 \pi} \frac{Z}{1-\psi} S\right)^{1 / 2}\left(\frac{3 \pi}{2} \frac{1-\psi}{Z v} \frac{p_{b}^{B}}{S}\right)^{1 / 6}
$$

If we assume perfect stick, the speed is, for typical Poisson's ratios, roughly, doubled.

\section{E. Deposition rate}

When $\mu_{b}$ is less than $\alpha$, a compression wave propagates into the flow to equilibrate the pressure difference across the interface. That is, the flow responds by decreasing its thickness and depositing particles to the bed. In this case, the rate of change of $b$ in Eq. (11) is equal to the speed of the pressure wave in the flow, Eq. (8), evaluated at $y=b$. With Eqs. (9) and (10),

$$
\frac{\partial b}{\partial t}=\left[\frac{p_{b}^{F}}{v}\left(2+\frac{G_{b}}{0.63}\right)\right]^{1 / 2}
$$

with

$$
G_{b}=\left[\frac{192 J^{2}(1-e)}{25 \hat{c} \pi^{3 / 2}(1+e)^{2}} \frac{1}{\mu_{b}^{3}}\right]^{3} .
$$

It might be more faithful to the physics if we employed the isentropic wave speed, rather than the isothermal, in that the former takes into account changes in temperature associated with rapid expansion and contraction of the granular gas. If employed, the factor of 2 in Eq. (20) would be replaced by $2+(4 / 3)(1+e) G_{b}$ and a somewhat higher wave speed would result.

\section{DISCUSSION AND RESULTS}

Before showing the results and the implications of the theory that we have outlined in the previous section, we summarize the main assumptions that we have employed and their physical justification. First, we have assumed that the erodible bed is the region in which long-lasting contacts, leading to a force network, are present, unlike the flow region above it, and that there the shear stress-to-pressure ratio has a characteristic value. The existence of elastic stresses associated with the development of a network of contacts, with the subsequent constancy of the stress ratio, at volume fractions larger than critical, has been revealed by numerical simulations [25]. Berzi and Jenkins [26] showed that identifying the erodible bed as the region in which elastic stresses develop explains the existence of the experimentally observed creeping flow in an erodible bed [27]. Second, we have assumed that, during erosion or deposition, the pressure and not the shear stress is discontinuous at the interface between the flow and the bed; as already mentioned, ad hoc discrete element simulations should be able to assess its validity. Third, we have assumed that the undisturbed pressure ahead of the moving pressure wave is hydrostatic. This seems plausible given that before the disturbance the stresses in the granular material had the time to equilibrate. Fourth, we have assumed that the relation between depth-averaged velocity, inclination, and flow thickness in unsteady flows is the same as in steady and fully developed flows. This assumption, as strong as it might be, is common in depth-averaged hydrodynamic models. It can be easily removed if the focus is not on a depth-averaged treatment of granular flows.

The rate of erosion or deposition in the depth-averaged mass balance is the difference between the particle velocity normal to the bed and the velocity of the surface of the bed. When they are equal, the surface of the bed is a material surface. One or the other may be constant. For example, in a steady flow down the surface of a heap in a cylindrical drum that rotates with constant angular velocity, the position of the bed is independent of the time, but the particle velocity normal to the bed varies with distance from the center [8,9]. In contrast, we focus here on situations in which the particle velocity at the bed is negligible, but the position of the bed is a function of time alone.

We employ Eqs. (19) and (20) for the speeds of the erosional and depositional interfaces, and calculate their displacement with time and the associated evolution of the particle flux in typical situations. The coefficient of restitution is 0.60 , the yield stress ratio is 0.40 , the volume fraction is 0.60 , the wall friction is 0.25 (as in [28]), the dimensionless cell width is 19, the coordination number is 5 , the dimensionless shear modulus is $10^{9}$, and Poisson's ratio is 0.20 . These are values appropriate for $0.53-\mathrm{mm}$ glass spheres flowing between glassy sidewalls at distance $1 \mathrm{~cm}$ apart, as in the experiments on flow initiation performed by Jop et al. [16]. For these values and typical flow depths and depth-average velocities, $v_{b}$ is a small fraction of the wave speed and may be neglected.

In uniform, unsteady situations, Eqs. (11) and (12) reduce to

$$
\frac{\partial h}{\partial t}=0
$$

and

$$
\begin{aligned}
\frac{\partial q}{\partial t}= & v(h-b) \cos \phi \\
& \times\left[\tan \phi-\mu_{b} \frac{p_{b}^{F}}{v(h-b) \cos \phi}-\frac{\mu_{w}}{W}(h-b)\right] .
\end{aligned}
$$

Equation (22) indicates that in this case the position of the free surface does not change. The system of Eqs. (23) and either (19) or (20) permits the determination of the evolution of the particle flux and the position of the bed in time, given the dependence of the slope on time.

We take the slope to change in a discontinuous way from the value $\tan \phi_{0}$ at $t=0$ to the value $\tan \phi_{1}$. A steady, uniform flow at an inclination angle of $\phi_{0}=28^{\circ}$ is disturbed by an increase (erosion) or a decrease (deposition) in the angle of inclination 


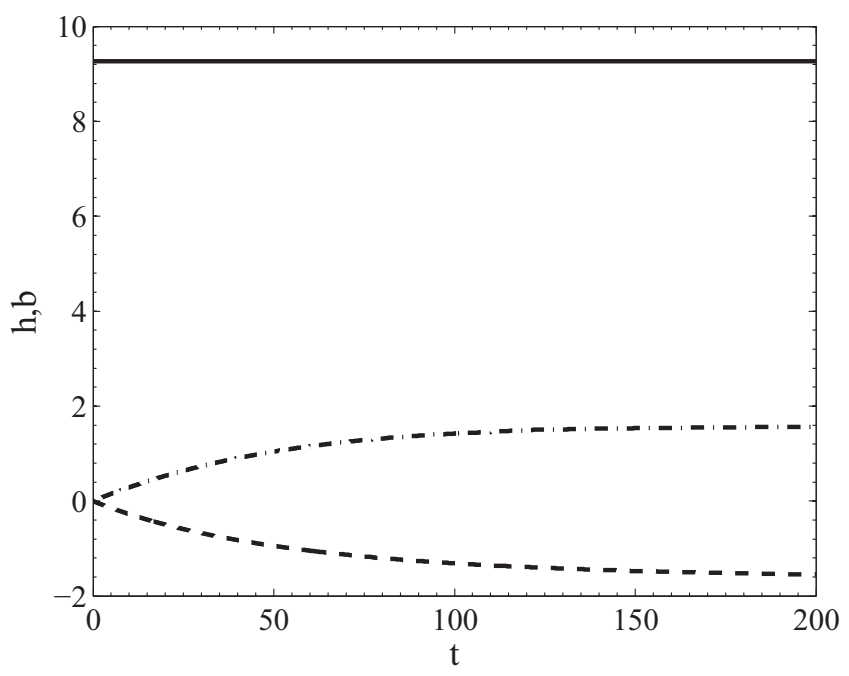

FIG. 1. Evolution of the position of the bed for erosion (dashed line, when $\phi_{0}=28^{\circ}$ and $\phi_{1}=29^{\circ}$ ) and for deposition (dot-dashed line, when $\phi_{0}=28^{\circ}$ and $\phi_{1}=27^{\circ}$ ). The solid line indicates the constant position of the free surface.

of $1^{\circ}$. To describe the subsequent adjustment of the bed and the evolution of the flow, the system of Eqs. (23) and (19) or (20) has been solved numerically using the function "ode113" implemented in MATLAB, with the initial values $b=0$ and $q=q_{0}$. The initial particle flux is determined from Eq. (14), with $\mu_{b}=\alpha$ and $h-b=W\left(\tan \phi_{0}-\alpha\right) / \mu_{w}$, obtained from Eq. (A4) with $s / p=\alpha$ (see the Appendix):

$$
\begin{aligned}
q_{0}= & \frac{2 v}{7}\left[\frac{\pi \cos \phi_{0}(1+e)}{8 J^{2} \tilde{G}}\right]^{1 / 2}\left(\tan \phi_{0}+\frac{5}{2} \alpha\right) \\
& \times\left(\frac{\tan \phi_{0}-\alpha}{\mu_{w}} W\right)^{5 / 2} .
\end{aligned}
$$

There are two characteristic times in the problem, one much shorter than the other. The shorter time scale is associated with the motion of the bed to the point at which $\mu_{b}=\alpha$ for the instantaneous value of $q$; the longer time scale is associated with the evolution of $q$. Figures 1 and 2 show, indeed, that the bed evolves simultaneously with the particle flux, and that it takes around 100 dimensionless time units to reach the final steady state. This is the time scale associated with the evolution of the flow.

We can calculate the order of magnitude of the time scale associated with the erosion process. Equation (19), with $p_{b}^{B}=$ $v(h-b) \cos \phi_{1}$, can be written as

$$
\frac{\partial b}{\partial t}=-A_{e}(h-b)^{1 / 6},
$$

with

$$
A_{e}=\left(\frac{11}{15 \pi} \frac{Z}{1-\psi} S\right)^{1 / 2}\left(\frac{3 \pi}{2} \frac{1-\psi}{Z} \frac{\cos \phi_{1}}{S}\right)^{1 / 6} .
$$

Then,

$$
h-b=\left[\left(\frac{\tan \phi_{0}-\alpha}{\mu_{w}} W\right)^{5 / 6}+\frac{5}{6} A_{e} t\right]^{6 / 5} .
$$

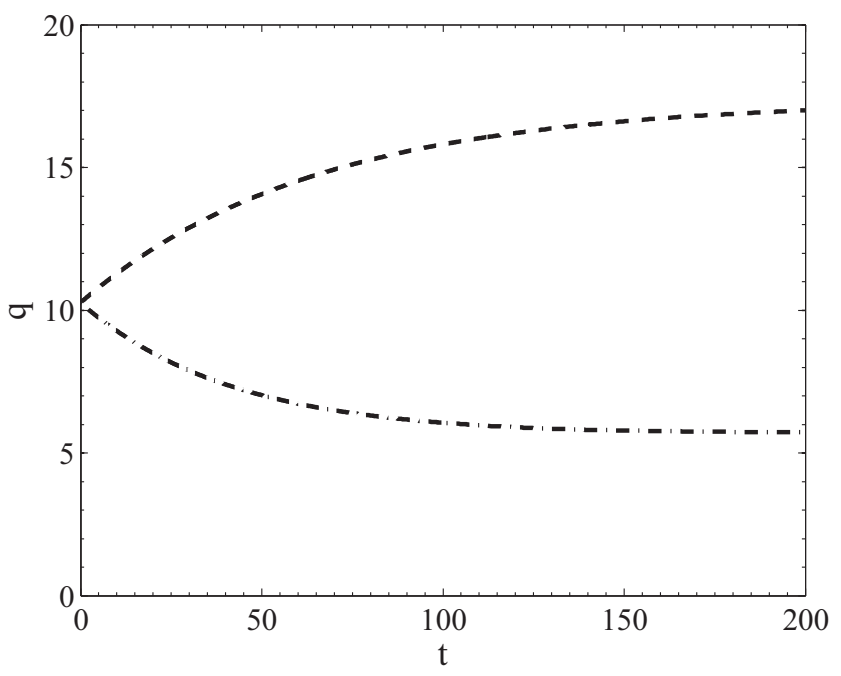

FIG. 2. Evolution of the particle flux for erosion (dashed line, when $\phi_{0}=28^{\circ}$ and $\phi_{1}=29^{\circ}$ ) and for deposition (dot-dashed line, when $\phi_{0}=28^{\circ}$ and $\phi_{1}=27^{\circ}$ ).

With this, we can evaluate the time, $t_{e}$, that it would take for the flow depth to reach the value $W\left(\tan \phi_{1}-\alpha\right) / \mu_{w}$ at the final steady state, if the process were governed by the pressure wave in the bed alone:

$$
t_{e}=\frac{6\left[\left(\tan \phi_{1}-\alpha\right)^{5 / 6}-\left(\tan \phi_{0}-\alpha\right)^{5 / 6}\right] W^{5 / 6}}{5 A_{e} \mu_{w}^{5 / 6}} .
$$

For deposition, Eq. (20), with $p_{b}^{F}=v(h-b) \cos \phi_{1}$, can be written as

$$
\frac{\partial b}{\partial t}=A_{d}(h-b)^{1 / 2}
$$

with

$$
A_{d}=\left[\left(2+\frac{G_{b}}{0.63}\right) \cos \phi_{1}\right]^{1 / 2}
$$

Then,

$$
h-b=\left[\left(\frac{\tan \phi_{0}-\alpha}{\mu_{w}} W\right)^{1 / 2}-\frac{A_{d}}{2} t\right]^{2} .
$$

In this case, the corresponding time is

$$
t_{d}=\frac{2\left[\left(\tan \phi_{0}-\alpha\right)^{1 / 2}-\left(\tan \phi_{1}-\alpha\right)^{1 / 2}\right] W^{1 / 2}}{A_{d} \mu_{w}^{1 / 2}} .
$$

Figure 3 shows the dependence of $t_{e}$ and $t_{d}$ on the final slope $\tan \phi_{1}$, assuming that $\tan \phi_{1} \leqslant k$ for a dense flow, when $\phi_{0}=28^{\circ}$. The relaxation time associated with the evolution of the flow is much greater than $t_{e}$ and $t_{d}$ (Fig. 2). This indicates that, at every step of integration, the position of the bed is determined by the condition $\mu_{b}=\alpha$; so, through Eq. (14), the instantaneous flow depth is a unique function of the particle flux. Then, with $h-b$ determined in terms of $q$, Eq. (23), which reduces to

$$
\frac{\partial q}{\partial t}=v(h-b) \cos \phi\left[\tan \phi-\alpha-\frac{\mu_{w}}{W}(h-b)\right],
$$

is sufficient to evaluate the evolution of $q$. 


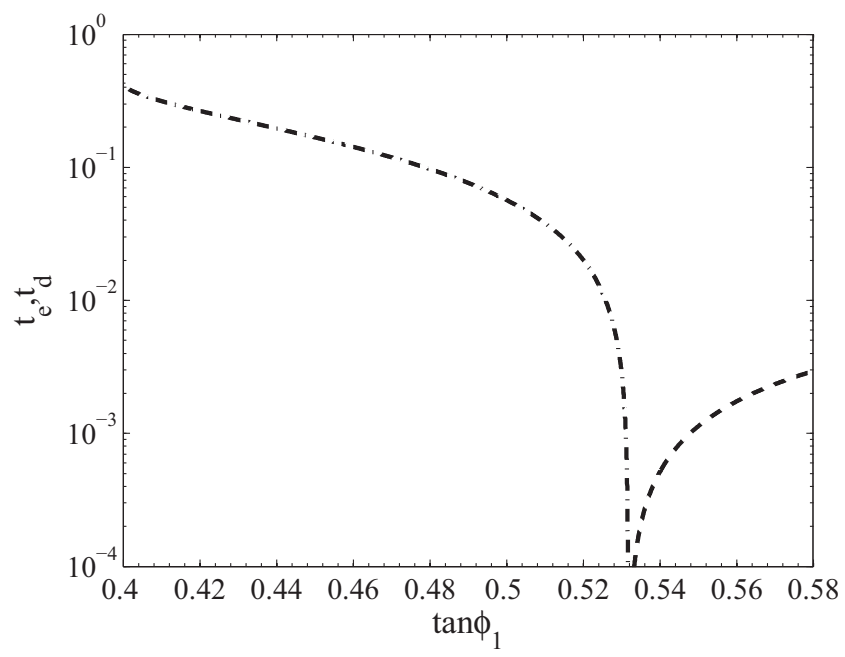

FIG. 3. Dependence of the time scales on the final slope, when the initial angle of inclination of the bed is $28^{\circ}: t_{e}$ (dashed line) and $t_{d}$ (dot-dashed line).

Figure 4 shows the comparisons between the present theory and the experiments of [16] on the initiation of flows of glass beads over erodible beds. We treat the initiation as an erosion process associated with an abrupt increase in the angle of inclination from $\tan \phi_{0}=\alpha$, corresponding to initial particle flux and flow depth equal to zero. The theory is able to reproduce the experimental results remarkably well, except at the largest angle. The lack of agreement for $\phi_{1}=29.5^{\circ}$ is due to the fact that $\tan \phi_{1}$ approaches the value $k=0.58$, which is the limit for the validity of Eq. (14) and the extended kinetic theory. Jop et al. [16] employ their phenomenological rheology and numerically integrate the resulting differential equations in three dimensions to obtain detailed features of

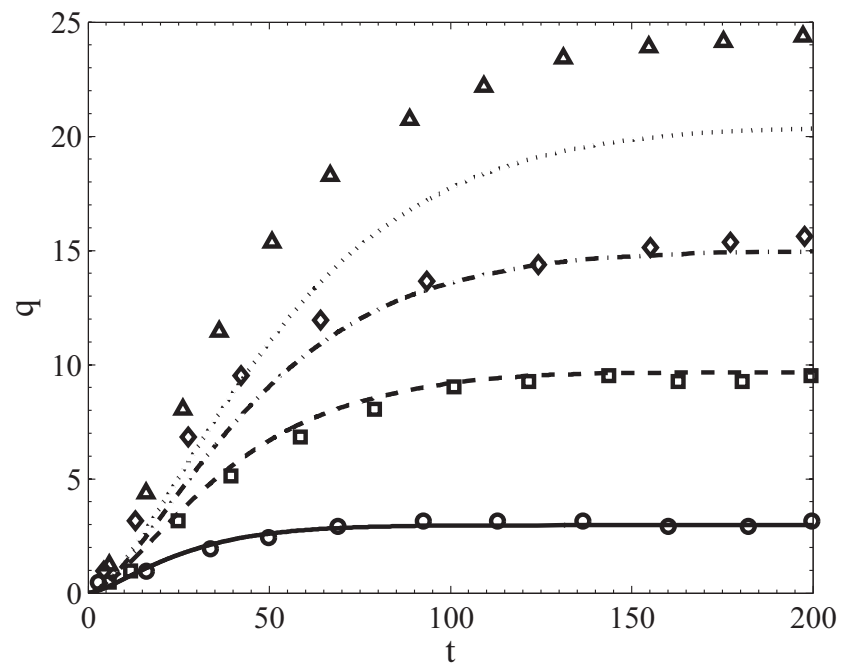

FIG. 4. Experimental (symbols, after Jop et al. [16]) against theoretical (lines) evolution of the particle flux starting from rest when $\phi_{1}$ is $26.1^{\circ}$ (circles and solid line), $28.0^{\circ}$ (squares and dashed line), $28.85^{\circ}$ (diamonds and dot-dashed line), and $29.5^{\circ}$ (triangles and dotted line). the evolving velocity profile that are in excellent agreement with their experimental measurements.

\section{CONCLUSIONS}

We wrote this paper to put forward a micromechanically based model for the rates of erosion and deposition at the interface between a dense, dry, inclined granular flow and an erodible bed. We assumed that the interface between the flowing grains and the bed moves with the speed of a pressure wave in the flow, for deposition, or with the speed a disturbance through the contacting spheres of the bed, for erosion. We employed the expressions for the rates of erosion and deposition to describe the evolution in time of the volume flow rate after an abrupt increase or decrease of the angle of inclination. We have distinguished between the instantaneous value of the stress ratio, $\mu_{b}$, at the base of the flow and the yield stress ratio, $\alpha$. We found that, as the flow evolves in either erosion or deposition, the pressure wave rapidly brings $\mu_{b}$ to $\alpha$. The results obtained agree with existing experiments on the initiation of flows of glass beads over erodible beds. The analysis that we have carried out puts the calculation of Jop et al. [16] into the context of existing treatments of erosion and deposition that introduce moving surfaces of discontinuity. Given our modeling of the flow, our conclusion is that the surface moves so quickly that the stress ratio at the base of the flow is always at its yield value and the instantaneous value of the particle flux is related to the instantaneous value of the flow depth by the same relation as in a uniform, steady flow. The latter relation and the momentum balance are therefore sufficient to describe the depth-averaged evolution of a granular flow over an erodible bed, without the necessity of introducing and modeling source and sink terms in the mass balance.

\section{APPENDIX: STEADY, UNIFORM, INCLINED FLOW}

As indicated earlier, we assume that in response to a change of inclination there is a very rapid adjustment through the depth of the flow, so that the depth averaged flow variables are related in the same way, but do not have the same values, as in a steady, uniform flow. In a steady, uniform, granular flow over an erodible bed confined between frictional sidewalls at an angle of inclination equal to $\phi[18]$, the momentum balances along the $x$ and $y$ direction read

$$
s^{\prime}=-v \sin \phi+2 \mu_{w} \frac{p}{W},
$$

and

$$
p^{\prime}=-v \cos \phi
$$

respectively, where a prime indicates a derivative with respect to $y$. Eliminating $d y$ between them and integrating gives

$$
\frac{s}{p}=\tan \phi-\mu_{w} \frac{p}{v W \cos \phi} .
$$

With $v$ approximately constant and $p=0$ at $y=h$, the integration of Eq. (A2) gives $p=v \cos \phi(h-y)$, so that

$$
\frac{s}{p}=\tan \phi-\mu_{w} \frac{h-y}{W} .
$$


The constitutive relation for the pressure and the shear stress are those of kinetic theory [24]

$$
p=f_{1} T,
$$

and

$$
s=f_{2} T^{1 / 2} u^{\prime},
$$

where $f_{1}=2 \nu G(1+e)$ and $f_{2}=8 \nu G J /\left(5 \pi^{1 / 2}\right)$. There, the function $G$ provides the dependence of the constant volume fraction on the angle of inclination when the flow is dense and the diffusion of fluctuating energy in the flow is negligible:

$$
G=\left[\frac{192 J^{2}(1-e)}{25 \hat{c} \pi^{3 / 2}(1+e)^{2}} \frac{1}{(s / p)^{3}}\right]^{3},
$$

in which $\hat{c}$ is a material coefficient of order 1 [29]. In order to carry out the integration for $u$, we approximate $G$ with its value $\tilde{G}$, at the average value $\alpha+\mu_{w}(h-y) /(2 W)$ of the stress ratio. Then, from Eqs. (A6), (A5), and (A3),

$$
u^{\prime}=\frac{f_{1}^{1 / 2}}{f_{2}} \frac{s}{p} p^{1 / 2}=\frac{f_{1}^{1 / 2}}{f_{2}} p^{1 / 2}\left(\tan \phi-\mu_{w} \frac{p}{v W \cos \phi}\right) .
$$

Using Eq. (A2) in Eq. (A8), and integrating with the boundary condition that $u=0$ when $p=p_{b}$,

$$
\begin{aligned}
u= & \frac{f_{1}^{1 / 2}}{v f_{2} \cos \phi}\left[\frac{2}{3} \tan \phi\left(p_{b}^{3 / 2}-p^{3 / 2}\right)\right. \\
& \left.-\frac{2}{5} \frac{\mu_{w}}{v W \cos \phi}\left(p_{b}^{5 / 2}-p^{5 / 2}\right)\right] .
\end{aligned}
$$

A further integration provides the depth-averaged flow velocity:

$$
\bar{u}=\frac{f_{1}^{1 / 2}}{v f_{2} \cos \phi} p_{b}^{3 / 2}\left(\frac{2}{5} \tan \phi-\frac{2}{7} \mu_{w} \frac{p_{b}}{v W \cos \phi}\right) .
$$

The shear rate at the bed, determined from Eq. (A8) when $p=p_{b}$, is, with Eq. (A10),

$$
u_{b}^{\prime}=\frac{5}{2} \frac{\bar{u}}{h-b}-\frac{2}{7} \frac{f_{1}^{1 / 2} p_{b}^{1 / 2}}{f_{2}} \mu_{w} \frac{h-b}{W} .
$$

Then, using Eqs. (A11) and (A5) in Eq. (A6), we obtain Eq. (13).
[1] N. Taberlet, P. Richard, E. Henry, and R. Delannay, Europhys. Lett. 68, 515 (2004)

[2] P. Jop, Y. Forterre, and O. Pouliquen, J. Fluid Mech. 541, 167 (2005).

[3] R. M. Iverson, M. E. Reid, M. Logan, R. G. LaHusen, J. W. Godt, and J. P. Griswold, Nature Geoscience 4, 116 (2010).

[4] A. Mangeney, O. Roche, O. Hungr, N. Mangold, G. Faccanoni, and A. Lucas, J. Geophys. Res. 115, 1 (2010).

[5] P. Richard, A. Valance, J.-F. Métayer, P. Sanchez, J. Crassous, M. Louge, and R. Delannay, Phys. Rev. Lett. 101, 248002 (2008).

[6] N. Jain, J. M. Ottino, and R. M. Lueptow, Phys. Fluids 14, 572 (2002).

[7] A. V. Orpe and D. V. Khakhar, J. Fluid Mech. 571, 1 (2007).

[8] J. Gray, J. Fluid Mech. 441, 1 (2001).

[9] N. Taberlet, P. Richard, and E. J. Hinch, Phys. Rev. E 73, 050301(R) (2006).

[10] J.-P. Bouchaud, M. E. Cates, J. Ravi Prakash, and S. F. Edwards, J. Phys. I 4, 1383 (1994).

[11] H. Capart and D. Young, J. Fluid Mech. 372, 165 (1998).

[12] L. Fraccarollo and H. Capart, J. Fluid Mech. 461, 183 (2002).

[13] M. Naaim, M. T. Faug, and F. Naaim-Bouvet, Survey in Geophysics 24, 569 (2003).
[14] Y. C. Tai and C. Y. Kuo, Acta Mech. 199, 71 (2008).

[15] R. M. Iverson, J. Geophys. Res. 117, 1 (2012).

[16] P. Jop, Y. Forterre, and O. Pouliquen, Phys. Fluids 19, 088102 (2007).

[17] J. T. Jenkins and C. Zhang, Phys. Fluids 14, 1228 (2002).

[18] J. T. Jenkins and D. Berzi, Granular Matter 12, 151 (2010).

[19] P. Digby, J. Appl. Mech. 48, 803 (1981).

[20] K. Walton, J. Mech. Phys. Solids 35, 213 (1987).

[21] I. Agnolin and J.-N. Roux, Phys. Rev. E 76, 061304 (2007).

[22] V. Garzó and J. W. Dufty, Phys. Rev. E 59, 5895 (1999).

[23] G. B. Whitham, Linear and Nonlinear Waves (Wiley, New York, 1974).

[24] J. T. Jenkins and D. Berzi, Granular Matter 14, 79 (2012).

[25] S. Chialvo, J. Sun, and S. Sundaresan, Phys. Rev. E 85, 021305 (2012).

[26] D. Berzi and J. T. Jenkins, Soft Matter 11, 4799 (2015).

[27] T. S. Komatsu, S. Inagaki, N. Nakagawa and S. Nasuno, Phys. Rev. Lett. 86, 1757 (2001).

[28] D. Berzi and J. T. Jenkins, Phys. Fluids 23, 013303 (2011).

[29] J. T. Jenkins, Granular Matter 10, 47 (2007). 